Bull. Austral. Math. Soc.

VOL. $56(1997) \quad[119-134]$

\title{
A ONE DIMENSIONAL ANALOGUE OF THE VORTICITY EQUATION
}

\author{
K. SRISKandarajah
}

\begin{abstract}
We study the qualitative properties of the one dimensional analogue of the Helmholtz vorticity advection equation. The second order hyperbolic equation has the unusual characteristic of disturbances propagating at infinite speed. The global solution for Goursat data is given in closed form. We also obtain qualitative results on the nodal curve where the solution is zero. A related perturbation problem is considered and solutions for small data are obtained. The forced vorticity equation admits a class of soliton solutions.
\end{abstract}

\section{INTRODUCTION}

The Helmholtz vorticity advection equation for the two dimensional flow of an incompressible ideal fluid, which describes the evolution of vorticity $\omega$, is given by

$$
\left(\frac{\partial}{\partial t}+u \frac{\partial}{\partial x}+v \frac{\partial}{\partial y}\right) \omega=0
$$

where $\mathbf{u}=(u(x, y, t), v(x, y, t))$ is the fluid velocity and $\omega=v_{x}-u_{y}$ is called the vorticity. We use the subscript notation to denote the partial derivatives and $\triangle$ to denote the two dimensional Laplacian operator. Conservation of mass is expressed by

$$
u_{x}+v_{y}=0
$$

The above two coupled equations are usually formulated in terms of the stream function $\psi$ that automatically satisfies conservation of mass. In terms of the stream function $\psi$, the flow variables are $u=-\psi_{y}, v=\psi_{x}, \omega=\psi_{x x}+\psi_{y y}=\Delta \psi$. The problem now is to solve the following nonlinear third order hyperbolic partial differential equation

$$
\left(\frac{\partial}{\partial t}-\psi_{y} \frac{\partial}{\partial x}+\psi_{x} \frac{\partial}{\partial y}\right) \Delta \psi=0
$$

\section{Received 16th Setpember, 1996}

I would like to express my gratitude to Professors R.H.J. Grimshaw and Klaus Ecker for their many helpful comments. I thank Dr. Alan Pryde and Dr. Bolis Basitt for a careful reading of the manuscript and for clarifying some points. I would like thank the referee for some useful comments on a previous version.

Copyright Clearance Centre, Inc. Serial-fee code: 0004-9729/97 \$A2.00+0.00. 
with the initial vorticity distribution $\omega(x, y, 0)$ specified in some region in the $x, y$ plane together with some boundary conditions. The vorticity equation implies that following the fluid particle trajectory defined by $\mathbf{x}_{t}=\mathbf{u}(\mathbf{x}, t)$ the vorticity is conserved and this is the Kelvin-Helmholtz vorticity conservation theorem for an inviscid fluid. Given the initial vorticity distribution one can solve for the stream function by inverting the Poisson equation $\Delta \psi=\omega$ and then find the velocity field and hence solve the advection equation to find the vorticity distribution at the next time step. This procedure is repeated until the desired time.

In [3] the authors model the three-dimensional vorticity equation by an integral differential equation. This is extended to viscous flow in [9]. A review of the malhematical theory and modeling of the three dimensional vorticity equation is found in [7]. The main difference in three dimensions is that vorticity can increase by stretching which in turn can cause singularities in velocity and vorticity to form in finite time. By contrast in two dimensions the solution exists globally with mild restrictions on the decay rate of the initial vorticity.

We would like to study a simpler problem in one space dimension that is similar in some respect to the vorticity advection equation to gain some understanding of the dynamics of the two dimensional vorticity equation. We consider

$$
\left(\frac{\partial}{\partial t}+u \frac{\partial}{\partial x}\right) u_{x}=0, t, x \in R
$$

subject to the boundary and initial conditions

$$
u(0, t)=g(t), u(x, 0)=u_{0}(x)=g(0)+\int_{0}^{x} f(\xi) d \xi .
$$

Here $u_{x}$ plays the role of vorticity. We are given $f$, the initial distribution of $u_{x}$. Equation (1) implies that $u_{x}$ is conserved along the curve $(x(t), t)$ defined by $x_{t}=$ $u(x, t)$.

In this paper our aim is to study the simpler equation (1). We follow the same steps outlined for the two dimensional problem. That is, for a fixed $(x, t)$ we

(i) find the trajectory $(\xi, t)$ for $\xi$ between 0 and $x$ of the fluid particles that reach $s$ at time zero,

(ii) establish the new distribution $u_{x}(\xi, t)=u_{x}(s, 0)=f(s)$,

(iii) integrate $u_{x}(\xi, t)$ from 0 to $x$ to find $u(x, t)$.

We assume the following hypotheses on $f$ and $g$ :

(Hf) $f$ is piecewise continuous and $|f(a)|<M$ for $a \in R, M>0$,

(Hg) $g$ is continuous. 
Writing (1) as a system of equations with $u_{1}=u, u_{2}=u_{x}$ gives

$$
u_{1 x}=u_{2}, u_{2 t}+u_{1} u_{2 x}=0 .
$$

This system of equations is in characteristic form and its characteristics are $t=$ constant and the curve $C$ given by $x_{t}=u_{1}(x, t)$. The characteristic speeds with which discontinuities of solutions propagate are the local speed $u$ along $C$ and infinite speed along $t=$ constant. The infinite speed of propagation is usually a feature of parabolic partial differential equations and not of hyperbolic equations.

The boundary condition $u(0, t)=g(t) \equiv 0$ makes the $t$ axis a characteristic. Therefore in the case $g \equiv 0$, the data (2) is specified on the two characteristics, the $t$ and $x$ axes. In the subsequent analysis we call $C$ the characteristic thus ignoring the trivial characteristic $t=$ constant. Along $C, u_{x}=u_{2}$ is constant and then integration gives $u(x, t)=\int_{0}^{x} u_{x}(\xi, t) d \xi$.

Integrating (1) from 0 to $x$ and using the boundary condition gives Burger's equation with a non-local source term

$$
\left(\frac{\partial}{\partial t}+u \frac{\partial}{\partial x}\right) u=\frac{d g}{d t}+\int_{0}^{x} u_{x}^{2} d x
$$

We show that solutions of (1) and (2) have no shocks and exist for all time in both directions. A similar equation occurs in gas flow through a nozzle. In $[5,6](3)$ with a right hand side $c(x) h(u)$ representing a moving local source is studied. It is found that shocks can develop in finite time. For further details see [6] and the references contained therein.

Define $u(x, t)$ to be a weak solution of (1) if $u$ is piecewise differentiable in $x$ for each fixed $t$ and satisfies (3) and the initial condition. This definition of weak solution allows us to include an initial condition $u_{0}$ which is only piecewise differentiable. It is shown that a weak solution of (1) is a classical solution except on the characteristics through the points where the initial function $f$ is not continuous and the discontinuity of the slope propagates with the local speed $u$.

As in [2], (1) and (2) could also be written as a first order nonlinear, non-local equation for $u_{x}(x, t)$ denoted by $v(x, t)$. Since $u(x, t)=g(t)+\int_{0}^{x} u_{x}(\xi, t) d \xi$ we get

$$
\frac{\partial v}{\partial t}+\left[g(t)+\int_{0}^{x} v(\xi, t) d \xi\right] \frac{\partial v}{\partial x}=0
$$

subject to the initial condition $v(x, 0)=f(x)$.

If $u(x, t)$ is a solution of (1) then

(i) $-u(-x, t)$ is a solution of (1) with $u_{0}(x), g(t)$ replaced by $-u_{0}(-x),-g(t)$ respectively, 
(ii) $u(x+L, t)$ solves (1) with initial condition $u_{0}(x+L)$ and boundary condition $u(L, t)=g(t)$,

(iii) $-u(x,-t)$ is a solution of (1) with $u_{0}(x), g(t)$ replaced by $-u_{0}(x),-g(-t)$ respectively.

Therefore without loss of generality we can assume $x \geqslant 0, t \geqslant 0$ in (1).

In the next section we analyse the case $g(t) \equiv 0$, which illustrates the method that is used to solve the problem for non-zero $g$ in a simpler setup. We give a plausible reason for the infinite speed of propagation for a compactly supported initial profile $u_{0}$. Suppose that $\lim _{x \rightarrow \infty} u(x, t)=c(t)$ for each fixed $t$. Then it follows from (3) that $d c / d t=\int_{0}^{\infty} u_{x}(x, t)^{2} d x>0, c(0)=0$. Hence $t c(t)>0$ and $c(t)$ is strictly increasing for all $t$. This means that $u(x, t)$ does not have compact support for any $t$ different from zero and this is what is meant by infinite speed of propagation. The assumptions made in deriving this result are proved valid in section 3 . From the observation in the previous paragraph and the last result a solution to (1) propagates to the right (left) with infinite speed if the zero boundary condition is to the left (right) of the support of $u_{0}$.

\section{Zero Boundary Condition}

In this section we study the characteristic curve $C$ through $(a, 0)$ defined by

$$
\frac{d x}{d t}=u(x(t), t), x(0)=a
$$

where $u(x, t)$ is a smooth solution to (1). Denote the solution to (1) by $x(a, t)$. Then formally we have

$$
\begin{aligned}
\frac{d}{d t} u_{x}(x(a, t), t) & =u_{t x}(x(a, t), t)+u_{x x}(x(a, t), t) x_{t}(a, t) \\
& =u_{t x}(x(a, t), t)+u(x(a, t), t) u_{x x}(x(a, t), t)=0 .
\end{aligned}
$$

Therefore for fixed $a$ we get $u_{x}(x(a, t), t)=$ constant $=f(a)$. Formally differentiating equation (5) with respect to the initial condition $a$ gives us

$$
\frac{d x_{a}}{d t}=u_{x}(x(t), t) x_{a}=f(a) x_{a}, x_{a}(0)=1 .
$$

The solution is

$$
x_{a}(a, t)=\int_{0}^{t} e^{u_{x}(x(a, \tau), \tau)} d \tau=e^{t f(a)} .
$$

Integration with respect to $a$ gives $x(a, t)=x(0, t)+\int_{0}^{a} x_{a}(s, t) d s=\int_{0}^{a} e^{t f(s)} d s$. So define $u(x(a, t), t)=x_{t}(a, t)=\int_{0}^{a} f(s) e^{t f(s)} d s$ and suppose that for every $(x, t)$ there is an $a$ such that $x(a, t)=x$. Then by reversing the arguments in this paragraph we get the the following theorem. 
THEOREM 1. Let a be implicitly defined by $x=x(a, t)=\int_{0}^{a} e^{t f(s)} d s$. Then $u(x, t)=\int_{0}^{a} f(s) e^{t f(s)} d s$ is the unique solution of (1) and (2) with $g \equiv 0$.

Before proving this theorem we observe that the solution exists for all time and this is in sharp contrast to the solutions of Burger's equation $u_{t}+u u_{x}=0$ which may develop shock in finite time or may lose uniqueness depending on the initial condition $u(x, 0)$. Suppose $\phi$ is an arbitrary function such that $\int_{-\infty}^{\infty} \phi(f(a)) d a$ is convergent. Then a change of variable gives that

$$
\int_{-\infty}^{\infty} \phi\left(u_{x}(x, t)\right) e^{-t u_{x}(x, t)} d x
$$

is a conserved quantity of (1).

Now we list several properties of $x(a, t)$ that are used in the proof of the theorem and later. The function $x(a, t)$ is infinitely differentiable in $t$ and its time derivatives are given by $\partial^{n} x(a, t) / \partial t^{n}=\int_{0}^{a} f^{n}(s) e^{t f(s)} d s$. Moreover, $x$ is one time more differentiable with respect to $a$ than is $f$. Hence we note that all the even partial derivatives of $x(a, t)$ with respect to $t$ are positive for $a>0$ making $x$ and all even derivatives of $x$ with respect to $t$ positive and convex. The hypothesis (Hf) implies that $a e^{-|t| M} \leqslant$ $\int_{0}^{a} e^{t f(s)} d s \leqslant a e^{|t| M}$. The positivity and the boundedness of the integrand shows that for a fixed $t$

(i) the Jacobian $\exp (t f(a))$ of the transformation from $(x, t)$ to $(a, t)$ is non-zero,

(ii) $x(a, t)$ is strictly increasing with $a$,

(iii) $\lim _{a \rightarrow \infty} x(a, t)=\infty$.

This in turn implies that the transformation defined by $x(a, t)=\int_{0}^{a} e^{t f(s)} d s$ has an inverse $a(x, t)$ for every fixed $t$, is one to one, onto and

$$
a_{x}=e^{-t f(a)}, a_{t}=-e^{-t f(a)} \int_{0}^{a} f(s) e^{t f(s)} d s .
$$

Therefore it follows that (i) characteristics do not intersect, (ii) every point $(x, t)$ is reached by the characteristic through $(a(x, t), 0)$. Hence the solution to $(1)$ is unique and exist for all time. Compare this with the solutions of Burger's equation which can develop a shock in finite time or lose uniqueness in the form of an expansion fan depending on the initial conditions. We may interpret $x(a, t), x_{t}(a, t), x_{t t}(a, t)$ as the position, velocity, and acceleration of the particle that was at $a$ initially. We collect a few more properties that are needed, in the following remark. These follow easily from the definition of $x$. 
REMARK 1.
(i) $x_{t}(a, t)=\int_{0}^{a} f(s) e^{t f(s)} d s=u(x(a, t), t)=u(x, t)$,
(ii) $x_{t a}(a, t)=f(a) e^{t f(a)}=u_{x}(x, t) x_{a}(a, t)=u_{x}(x(a, t), t) e^{t f(a)}$,
(iii) $x_{t t}(a, t)=\int_{0}^{a} f^{2}(s) e^{t f(s)} d s=u_{x}(x(a, t), t) x_{t}(a, t)+u_{t}(x(a, t), t)$.

Proof: It is easy to see that $u$ satisfies the boundary and initial conditions. Now to verify $u(x, t)$ satisfies the differential equation we differentiate $u_{x}(x(a, t), t)=f(a)$, given by (i), with respect to $t$ and it follows that $0=u_{t x}(x(a, t), t)+u_{x x}(x(a, t), t) x_{t}(a, t)$ $=u_{t x}+u u_{x x}$ everywhere except on the characteristics through the points $(a, 0)$ where $f$ is discontinuous. If $f$ were continuous then the solution would be classical. The uniqueness of the solution follows easily from the method of characteristics and the proof is therefore omitted.

We have shown in the proof of Theorem 1 that a classical solution is a weak solution and a weak solution is a classical solution except on the characteristics through the discontinuities of $u_{0}^{\prime}=f$.

The asymptotic behaviour of $x(a, t), x_{t}(a, t), x_{t t}(a, t)$ for fixed $a$ and large $t$ are governed by the sign of $f$ in $(0, a)$. To quantify we define the following:

$$
\begin{gathered}
\triangle_{+}(a)=\{s \in(0, a): f(s)>0\}, \triangle_{-}(a)=\{s \in(0, a): f(s)<0\} \\
\triangle_{0}(a)=\{s \in(0, a): f(s)=0\} \\
\bar{a}=\inf \left\{a>0:\left|\triangle_{-}(a)\right|>0 \text { and }\left|\triangle_{+}(a)\right|=0 \text { or }\left|\triangle_{+}(a)\right|>0 \text { and }\left|\triangle_{-}(a)\right|=0\right\} .
\end{gathered}
$$

Therefore $\bar{a}$ is essentially the first time $f$ switches sign. Here and elsewhere, the measure of a set $S$ is denoted by $|S|$. The different possibilities for $x(a, t), x_{t}(a, t)$ for a fixed $a>0$ are listed below.

\section{LEMMA 1 .}

(i) As a function $t, x(a, t)$ is convex and positive, and $x_{t}(a, t)$ is strictly increasing in $t$ for all $t$.

(ii) If $\left|\triangle_{+}(a)\right|>0$ and $\left|\triangle_{-}(a)\right|>0$ then $\lim _{t \rightarrow \pm \infty} x_{t}(a, t)= \pm \infty$.

(iii) If $\left|\triangle_{+}(a)\right|=0$ and $\left|\triangle_{-}(a)\right|>0$ then $\lim _{t \rightarrow \infty} x_{t}(a, t)=0, \lim _{t \rightarrow \infty} x(a, t)=$ $\left|\triangle_{0}(a)\right|$.

(iv) If $\left|\triangle_{+}(a)\right|>0$ and $\left|\triangle_{-}(a)\right|=0$ then $\lim _{t \rightarrow-\infty} x_{t}(a, t)=0, \lim _{t \rightarrow-\infty} x(a, t)=$ $\left|\triangle_{0}(a)\right|$.

Proof: The proof of (i) follows from the integrand of both $x(a, t)$ and $x_{t t}(a, t)$ 
being positive. If $\left|\triangle_{+}(a)\right|>0$ then for $t>0$ we have

$$
\begin{aligned}
x_{t t}(a, t) & =\int_{0}^{a} f^{2}(s) e^{t f(s)} d s=\int_{\triangle_{+}(a)} f^{2}(s) e^{t f(s)}+\int_{\triangle_{-}(a)} f^{2}(s) e^{t f(s)} \\
& \geqslant \int_{\Delta_{+}(a)} f^{2}(s) e^{t f(s)} \geqslant \int_{\Delta_{+}(a)} f^{2}(s) .
\end{aligned}
$$

This shows that in general

$$
x_{t t}(a, t) \geqslant \operatorname{Min}\left\{\int_{\triangle_{+}(a)} f^{2}(s), \int_{\triangle_{-}(a)} f^{2}(s)\right\} \text { for all } t .
$$

Similarly it can be shown that if $\left|\Delta_{-}(a)\right|>0$ then $x_{t}$ tend to infinity as $t$ goes to negative infinity. If $\left|\triangle_{+}(a)\right|=0$ then all the integrals are over $\triangle_{-}(a)$ and the integrands are bounded. We use the Lebesque dominated convergence theorem to show the limits exist as $t$ goes to infinity. Since $\left|f(s) e^{t f(s)}\right| \leqslant|f(s)|$ for $s \in \triangle_{-}(a), t \geqslant 0$,

$$
\lim _{t \rightarrow \infty} \int_{\Delta_{-}(a)} f(s) e^{t f(s)}=\int_{\Delta_{-}(a)} \lim _{t \rightarrow \infty} f(s) e^{t f(s)}=0 .
$$

Similarly

$$
\lim _{t \rightarrow \infty} \int_{\Delta_{-}(a)} e^{t f(s)}=\int_{\Delta_{-}(a)} \lim _{t \rightarrow \infty} e^{t f(s)}=0
$$

Now

$$
x(a, t)=\int_{0}^{a} e^{t f(s)} d s=\int_{\Delta_{-}(a)} e^{t f(s)}+\int_{\Delta_{0}(a)} e^{t f(s)} d s .
$$

Taking the limit as $t$ going to infinity gives $\lim _{t \rightarrow \infty} x(a, t)=\left|\triangle_{0}(a)\right|$. Similar arguments lead to $\lim _{t \rightarrow \infty} x_{t}(a, t)=x_{t t}(a, t)=0$ and to the counterparts for the case when $\left|\triangle_{-}(a)\right|=$ 0 . Combining the different cases now shows the parts (ii),(iii), and (iv).

Lemma 1 shows that $x_{t}(a, t)$ is strictly increasing in $t$ and has at most one zero for each fixed $a \in R$. This means that along each characteristic $x(a, t)$ the value $u(x(a, t), t)=x_{t}(a, t)$ is a strictly increasing function of $t$ and the behaviour at $t= \pm \infty$ depends on $\triangle_{+}(a), \Delta_{-}(a)$ as stated in the lemma.

Therefore we can define $t(a)$ as the unique $t$ such that $x_{t}(a, t(a))=0$ if $\left|\triangle_{+}(a)\right|>$ 0 and $\left|\triangle_{-}(a)\right|>0, t(a)=\infty$ if $\left|\triangle_{+}(a)\right|=0$ and $\left|\triangle_{-}(a)\right|>0$ and $t(a)=-\infty$ if $\left|\Delta_{+}(a)\right|>0$ and $\left|\Delta_{-}(a)\right|=0$. It follows from the uniqueness of $t$ that (i) $t(a)=$ 0 if and only if $u_{0}(a)=0$, (ii) $t(a) u_{0}(a)<0$, (iii) $t(\bar{a}) \operatorname{sgn}\left(u_{0}(\bar{a})\right)=\infty$ and the domain of $t$ is $(\bar{a}, \infty)$. In [1] and [8] the number of points in the zero set $\{x: u(x, t)=0\}$ of $u$ is defined as the lap number. It is shown using the maximum principle that for 
solutions of parabolic partial differential equations the lap number is a non-increasing function of time. In our case we show that for a bounded initial function the lap number is zero or one as $t$ goes to positive or negative infinity. The lap number in our case is the same as the number of points in the set $\{a: t(a)=t\}$ when the latter is defined.

The significance of $t(a)$ is that if we set $x=x(a, t)$ then $t$ is a function of $x$ parametrised by $a$ and $u(x, t(x))=0$. Here we have used the same $t$ to denote both a function of $a$ and a function of $x$. Therefore we can track the zeros of the solution $u(x, t)$ in space and time obtaining the nodal curve of $u$ which is defined by $\{(x(a, t(a)), t(a)): a \in(\bar{a}, \infty)\}$ and is studied further in the next section. He and Moodie refer to the nodal curve of $u$ as a sonic line in [5].

\section{Nodal Curves of $u$}

It is found easier to study the qualitative properties of the function $t(a)$ through a differential equation satisfied by $t(a)$. We derive this differential equation from the condition $x_{t}(a, t)=0$ for all $a$. Differentiating with respect to $a$ we get $x_{t a}(a, t)+$ $x_{t t}(a, t) d t / d a=0$. This gives

$$
\frac{d t}{d a}=\frac{-f(a) e^{t f(a)}}{\int_{0}^{a} f^{2}(s) e^{t f(s)} d s}
$$

subject to the initial condition

$$
t\left(a_{0}\right)=t_{0} \text { where } u\left(a_{0}, t_{0}\right)=0 \text { or } t(\bar{a})=\operatorname{sgn}\left(u_{0}(\bar{a})\right) \infty .
$$

\section{REMARK 2.}

(i) $u_{0}(a)$ and $t(a)$ have the opposite sign and the same critical points in the domain of $t$,

(ii) $\operatorname{supp} d t / d a=\operatorname{supp} f$.

LEMmA 2. The solution to (8) exists on $(\bar{a}, \infty)$ where $\bar{a}=\inf \left\{a>0:\left|\triangle_{-}(a)\right|>\right.$ 0 and $\left|\triangle_{+}(a)\right|=0$ or $\left|\triangle_{+}(a)\right|>0$ and $\left.\left|\triangle_{-}(a)\right|=0\right\}$. If in addition $\lim _{a \rightarrow \infty} u_{0}(a)$ exists then $t(a)$ is bounded for $a \in(\bar{a}+\varepsilon, \infty)$ for any $\varepsilon>0$.

PRoOF: We showed in the proof of Lemma 1 in the last section that if $\left|\triangle_{+}\left(a_{0}\right)\right|>$ $0,\left|\triangle_{-}\left(a_{0}\right)\right|>0$ then

$$
x_{t t}(a, t) \geqslant \operatorname{Min}\left\{\int_{\triangle_{+}\left(a_{0}\right)} f^{2}(s), \int_{\triangle_{+}\left(a_{0}\right)} f^{2}(s)\right\}=B\left(a_{0}\right) \text { for all } t \text { and } a \geqslant a_{0} .
$$

It follows that $d t / d a$ is bounded above by $M e^{|t| M} / B\left(a_{0}\right)$ for any $\bar{a}<a_{0} \leqslant a$. This implies global existence of solutions on $(\bar{a}, \infty)$. 
To prove the second part suppose $t$ and $d t / d a$ are positive in $\left(a_{0}, a\right)$. Then it follows from the differential equation that $f$ is negative in $\left(a_{0}, a\right)$ and implies

$$
\frac{d t}{d a} \leqslant-\frac{f(a) e^{t f(a)}}{B\left(a_{0}\right)} \leqslant-\frac{f(a)}{B\left(a_{0}\right)} .
$$

This gives

$$
t(a) \leqslant t\left(a_{0}\right)+\frac{\left|u_{0}(a)-u_{0}\left(a_{0}\right)\right|}{B\left(a_{0}\right)} .
$$

Letting $a$ go to infinity shows that $t$ is positive and bounded. A similar argument shows that $t$ is bounded from below and hence proves the lemma.

Connectedness of the graph $(a, t(a))$ shows that if $u_{0}$ has a non-trivial zero then the zeros of $u(x, t)$ can be traced to the zeros of the initial profile $u_{0}$. Since there is a one to one correspondence between the $(a, t)$ plane and the $(x, t)$ plane we can convert the nodal curve of $u$ from one plane to the other; it merely changes the domain of $t$. The domain of $t$ as a function of $x$ is $\left(\lim _{t \rightarrow \pm \infty} x(\bar{a}, t), \infty\right)=\left(\left|\triangle_{0}(\bar{a})\right|, \infty\right)$. That is, the nodal curve in the $(x, t)$ plane is a horizontally strained version of the nodal curve in the $(a, t)$ plane having the zeros of $u_{0}$ as the zeros of both functions.

To illustrate the effectiveness of $t(a)$ in predicting the qualitative behaviour of the evolution of $u(x, t)$ we look at an initial condition $u_{0}(x)$ with compact support.

Consider an initial condition $u_{0}$ with compact support $[0, L]$. Then from the definition $\bar{a} \in(0, L)$. We can assume without loss of generality that $u_{0}(\bar{a})>0$. The remark preceding the lemma in the last section implies $(i) \lim _{a \rightarrow \bar{a}} t(a)=-\infty,(i i) t(a)=$ 0 for $a \geqslant L$. This means that for any non-zero $t_{1}, u\left(x, t_{1}\right)$ is not zero for $x \geqslant x\left(L, t_{1}\right)$ since $t^{-1}\left(t_{1}\right)<L$. Therefore $u(x, t)$ has no compact support for any non-zero $t$. More can be said about the behaviour of $u(x, t)$ for large $x$ by computing $u(x, t)$ given in Theorem 1. $u(x, t)=x_{t}(L, t)$ for $x \geqslant x(L, t)$ and $x_{t}(L, t)$ is a strictly increasing function of time. In other words, $u(x, t)$ loses its compact support for any non-zero $t$ in a mild way by maintaining compact support for $u_{x}(., t)$ for all $t$. Also observe that $\lim _{t \rightarrow \pm \infty}\left|\operatorname{supp}\left\{u_{x}(x, t)\right\}\right|=\infty$. By specialising the above analysis for a non-negative initial profile $u_{0}$ with compact support, we conclude that $u(x, t)$ is positive for $t>0$ and changes sign once for $t<0$. From the symmetry of (1) it follows that if the zero boundary condition is to the left (right) of the compact support of the initial condition then $u(x, t)$ propagates with infinite speed to the right (left).

\section{Non Zero Boundary Condition}

We consider (1) with a general boundary condition. That $g \neq 0$ makes the $t$ axis no longer the characteristic $x(0, t)$ through $(0,0)$ and introduces new difficulties. We 
define $x(0, t)=\gamma(t)$. The characteristic through $(a, 0)$ is given by $x(a, t)=x(0, t)+$ $\int_{0}^{a} e^{t f(s)} d s=\gamma(t)+\int_{0}^{a} e^{t f(s)} d s$. It turns out that it is easier to study $b(t)=a(0, t)$ than $\gamma(t)$. The function $b(t)$ has the property that the characteristic $x(b(t), t)$ through $(b(t), 0)$ goes through $(0, t)$. That is, we follow the characteristic starting from the $t$ axis where we know the value of $u$, hence the slope of the characteristic, and this intersects the $x$ axis at $b(t)$.

We derive a differential equation for $b$ from the identity $x(b(t), t)=0$. Differentiation with respect to $t$ gives

$$
x_{t}(b(t), t)+x_{a}(b(t), t) \frac{d b}{d t}=0
$$

and

$$
x_{t}(b(t), t)=u(x(b(t), t))=u(0, t)=g(t)
$$

Therefore

$$
e^{t f(b)} \frac{d b}{d t}+g(t)=0
$$

The initial condition for $b$ is $b(0)=0$. The existence and uniqueness of solutions to the differential equation (11) is established.

If $f$ is continuous and bounded then the proof of uniqueness, existence and boundedness of $b$ follows easily. To prove the same when $f$ is only piecewise continuous needs some care. Indeed no solution to the simple differential equation $\operatorname{sgn}(b) d b / d t+1=0, b(0)=0$ can be continued to the right of time zero. However across a discontinuity of $f$ the coefficent of the slope of $b$ is positive and hence $b$ can be continued past a discontinuity of $f$ in both directions. In this way we obtain a unique, bounded and piecewise differentiable function $b$ that exists for all time. This is stated as a lemma.

Lemma 3. Suppose $g$ is continuous and $f$ is piecewise continuous and bounded. Then the solution to (9) exists on all of $R$, and is unique, piecewise continuous and bounded.

We can now obtain a formula for $x(a, t)$ in terms of $b$ and $f$. As before

$$
x(a, t)=x\left(a_{1}, t\right)+\int_{a_{1}}^{a} x_{a}(s, t) d s=x\left(a_{1}, t\right)+\int_{a_{1}}^{a} e^{t f(s)} d s .
$$

If $a_{1}=b(t)$ then

$$
x(a, t)=\int_{b(t)}^{a} e^{t f(s)} d s .
$$

This leads us to the following remarks on the properties of $x(a, t)$ that we shall need later. 
REMARK 3.

(i) $x(b(t), t)=0, x_{t}(b(t), t)=g(t)$ for all $t$,

(ii) $x_{t}(a, t)=x_{t}(b(t), t)+\int_{b(t)}^{a} x_{t a}(s, t) d s=g(t)+\int_{b(t)}^{a} f(s) e^{t f(s)} d s=u(x, t)$,

(iii) $x_{t t}(a, t)=g^{\prime}(t)+g(t) f(b(t))+\int_{b(t)}^{a} f^{2}(s) e^{t f(s)} d s$.

Since $b$ is bounded it follows from (11) that for a fixed $t$ the limits $\lim _{a \rightarrow \pm \infty} x(a, t)=$ $\pm \infty$ and $x(a, t)$ is strictly increasing as a function of $a$. Therefore the coordinate transformation $(a, t)$ to $(x, t)$ defined by $x=\int_{b(t)}^{a} e^{t f(s)} d s$ is one-one and onto. As before $u$ defined by (ii) of Remark 3 is the unique solution to (1) and (2). The above discussion leads us to the main theorem.

THEOREM 2. Let $a$ be implicitly defined by $x=\int_{b(t)}^{a} e^{t f(s)} d s$ where $b$ is the solution of (10). Then $u(x, t)=g(t)+\int_{b(t)}^{a} f(s) e^{t f(s)} d s$ is the unique solution to (1) and (2).

REMARK 4. We can estimate the growth rate of $u$ for fixed $t$ from the formula given above. The mean value theorem for integrals gives $|(u(x, t)-g(t)) / x| \leqslant M$ where $M$ is the bound for $f$. Therefore the growth rate of $u$ is at most linear in $x$ for each fixed $t$.

Another way to look at $b$ is to choose any $b$ with $b(0)=0$ and define $g$ by $(10)$. Then we can control the shape of $x(a, t)$ through $g$ and hence $u$ on the characteristics. The separation $x\left(a_{2}, t\right)-x\left(a_{1}, t\right)$ of the characteristics through $a_{1}, a_{2}$ is still controlled by the sign of $f$ in $\left(a_{1}, a_{2}\right)$ and the choice of $b$ has no influence on this. We shall use these remarks to help us select $f$ and $h$ in order to have a soliton solution $u_{x}$ of $u_{x t}+u u_{x x}=h\left(u_{x}\right)$ and $(2)$.

\section{Perturbation Equation}

Consider

$$
\left(\frac{\partial}{\partial t}+\varepsilon u \frac{\partial}{\partial x}\right) u_{x}=0, \varepsilon \in R
$$

subject to the boundary and initial conditions (2). In the limit $\varepsilon$ going to zero formally we get the linear wave equation $u_{x t}=0$. The solution is $u(x, t ; 0)=u_{0}(x)+g(t)-g(0)=$ $g(t)+\int_{0}^{x} f(s) d s$. Therefore we expect that if we denote the solution of (12) and (2) by $u(x, t ; \varepsilon)$ then $\lim _{\varepsilon \rightarrow 0} u(x, t ; \varepsilon)=u_{0}(x)+g(t)$. We show that this is indeed the case.

Suppose $u(x, t ; \varepsilon)$ is a solution of (12). Then $v$ defined by $\varepsilon v=u$ satisfies (1) and (2) with $u_{0}$ replaced $\varepsilon u_{0}$ and $g$ replaced by $\varepsilon g$. That is what we need to solve (1) with small data $\varepsilon f, \varepsilon g$. To compute $v(x, t ; \varepsilon)$ we follow the steps given in the Theorem 2 . 
LEMma 4. With the notations in the previous paragraph for $t, a$ fixed

(i) $b=-\varepsilon\left(\int_{0}^{t} g(s) d s\right)+O\left(\varepsilon^{2}\right)$,

(ii) $x=x(a, t ; \varepsilon)=a+O(\varepsilon)$,

(iii) $v(x, t ; \varepsilon)=\varepsilon\left\{g(t)+\int_{0}^{x} f(s) d s\right\}+O\left(\varepsilon^{2}\right)$.

Proof: Since $b$ satisfies

$$
e^{\varepsilon t f(b)} \frac{d b}{d t}+\varepsilon g(t)=0, b(0)=0,
$$

$b(t)$ is implicitly given by

$$
\begin{aligned}
b(t) & =-\varepsilon\left\{\int_{0}^{t} g(s) d s+\int_{0}^{t} g(s)\left(e^{-\varepsilon s f(b(s))}-1\right) d s\right\} \\
& =-\varepsilon\left\{\int_{0}^{t} g(s) d s+R\right\} \text { where } R=\int_{0}^{t} g(s)\left(e^{-\varepsilon s f(b(s))}-1\right) d s .
\end{aligned}
$$

We show $R / \varepsilon$ is bounded. Note

$$
\left|g(s)\left(e^{-\varepsilon s f(b(s))}-1\right)\right| \leqslant\left|g(s) \varepsilon s f(b(s)) e^{-\varepsilon \theta s f(b(s))}\right| \leqslant|s g(s)| M e^{|\varepsilon s| M} .
$$

We used the Mean Value Theorem and that $f$ is bounded in the last step. Therefore we have $R=O(\varepsilon)$ which proves (i). We prove (ii) by the same arguments used in (i) after writing

$$
\begin{aligned}
x(a, t) & =\int_{b(t)}^{a} e^{\varepsilon t f(s)} d s \\
& =a-b(t)+\int_{b(t)}^{a}\left(e^{\varepsilon t f(s)}-1\right) d s .
\end{aligned}
$$

That is, $x=x(a, t)=a+O(\varepsilon)$. Similarly for $v$ we find

$$
\begin{aligned}
v(x(a, t), t) & =\varepsilon g(t)+\int_{b(t)}^{a} \varepsilon f(s) e^{\varepsilon t f(s)} d s \\
& =\varepsilon\left\{g(t)+\int_{b(t)}^{a} f(s) d s+\int_{b(t)}^{a} f(s)\left(e^{\varepsilon t f(s)}-1\right) d s\right\} \\
& =\varepsilon\left\{g(t)+\int_{b(t)}^{a} f(s) d s+O(\varepsilon)\right\}=\varepsilon\left\{g(t)+\int_{0}^{a} f(s) d s+O(\varepsilon)\right\} .
\end{aligned}
$$

Therefore we have proved the following theorem.

Theorem 3. Let $u(x, t ; \varepsilon)$ be the unique solution of (12) and (2). Then for fixed $(x, t)$ we have $\lim _{\varepsilon \rightarrow 0} u(x, t ; \varepsilon)=g(t)+u_{0}(x)-g(0)$. 


\section{Soliton Solutions}

In this section we look for a soliton solution $u_{x}(x, t)$ (see [4]) of

$$
\left(\frac{\partial}{\partial t}+u \frac{\partial}{\partial x}\right) u_{x}=h\left(u_{x}\right)
$$

using the methods developed in the previous sections. We have been able to control the shape of the characteristics by $b$ through $g$ and the separation between two characteristics depends only on the sign of $f$ between the points. In the case of $h=0, u_{x}$ is constant on the characteristics; hence the the profile of $u_{x}(x, t)$ is spreading between the characteristics coming from the positive regions of $f$ and shrinking between the characteristics coming from the negative regions of $f$ as time increases. With $h$ not identically zero we have now a means of controlling the the value of $u_{x}(x, t)=u_{x}(x(a, t), t)$ on the characteristic through $(a, 0)$ with time. This is given by the solution to the ordinary differential equation

$$
\frac{d v}{d t}=h(v), v(0)=f(a)
$$

Therefore there is a possibility for a soliton type solution (13) for $u_{x}(x, t)$ for some choice of $f, b$ and $h$. We look for a solution $u$ of $(13)$ so that $u_{x}(x, t)$ for each fixed $t$ is a translation of $f$ and decays to zero at great distances.

In $[2]$ the connection between the solutions of (13) and a one dimensional non-local wave equation is established by a nonlinear transformation of a dependent variable. The solvability of (13) is used to obtain solutions to the nonlinear one dimensional wave equation. The solution $u_{x}(x(a, t), t)$ of (13) is implicitly given by

$$
\int_{f(a)}^{u_{x}(x, t)} \frac{d v}{h(v)}=t
$$

Then (6) gives $x_{a}(a, t)=\int_{0}^{t} e^{u_{x}(x(a, \tau), \tau)} d \tau$. Therefore the transformation from $(x, t)$ to $(a, t)$ is given by $x=x(a, t)=\gamma(t)+\int_{0}^{a} x_{a}(s, t) d s$ and $u(x, t)=d \gamma / d t+\int_{0}^{a} x_{a t}(s, t) d s$ where $x(0, t)=\gamma(t)$ is the characteristic through the origin. We have therefore proved the following existence and uniqueness theorem.

THEOREM 4. Let $a$ be implicitly defined by

$$
x=x(a, t)=\gamma(t)+\int_{0}^{a}\left\{\int_{0}^{t} e^{u_{x}(x(s, \tau), \tau)} d \tau\right\} d s .
$$

Then $u(x, t)=\gamma^{\prime}(t)+\int_{0}^{a} x_{a t}(s, t) d s$ is the unique solution of (13) and (2) where $u_{x}(x(a, t), t)$ is the solution of (14) and $\gamma$ and $g$ are connected by $u(0, t)=g(t)$. 
REMARK 5. Theorem 5 generalises Theorems 1 and 2 with $\gamma$ and $b$ connected by $\gamma(t)=x(0, t)=x(b, t)+\int_{b}^{0} x_{a}(s, t) d s=\int_{b}^{0} x_{a}(s, t) d s$.

As in [2], (13) has the following class of conserved quantities. Let $\phi$ be any function so that $\int_{-\infty}^{\infty} \phi(f(a)) d a$ is convergent. Then by change of variable this integral transforms to

$$
\int_{-\infty}^{\infty} \frac{\phi\left(u_{x}(x, t)\right)}{x_{a}(a(x, t), t)} d x
$$

and hence is conserved.

We now resume the search for $f$ and $h$ so that (13) admits a soliton solution for $u_{x}(x, t)$. Suppose $f$ is positive, bell shaped and decays to zero far away. Let $f$ satisfy

$$
f>0, f^{\prime}(0)=0, x f^{\prime}(x)>0 \text { for } x \neq 0 \text { and } \lim _{|x| \rightarrow \infty} f(x)=0 .
$$

Letting $x$ go to infinity in (13) gives $h(0)=0$ for (13) to have a soliton solution. If $f(0)$ is a zero of $h$ then $f(0)$ is an equilibrium solution of $(14)$ and $u_{x}(\gamma(t), t)=f(0)$ for all $t$. Since $f$ is positive the characteristics are spreading with time so we need $h$ to be negative in $(0, f(0))$. In addition the solutions given by (14) exist globally for intial values in $[0, f(0)]$ if the zeros of $h$ are simple. Therefore choose $h(v)=$ $v(v-f(0)) h_{2}(v)$ with $h_{2}$ positive in $[0, f(0)]$. Since $\alpha u(x, \alpha t)$ solves (13) with a right hand side $\alpha^{2} h(u / \alpha)$, we can take $f(0)=1$ without loss of generality.

The functions $f$ and $h_{2}$ are to be chosen so that $u_{x}(x, t)=f(x-\gamma(t))$ is a solution. Substituting $u(x, t)=F(x-\gamma(t))+\gamma^{\prime}(t)$ where $F$ is the antiderivative of $f$ with $F(0)=0$ in (14) gives

$$
F(x) f^{\prime}(\xi)=h(f(x))
$$

The general solution is $F(x)=c e^{H_{1}(f(x))}$ where $c$ is a constant and $H_{1}$ is an antiderivative of $v / h(v) . F(0)=0$ implies that $H_{1}(v)$ goes to negative infinity as $v$ approaches 1 from the left. Therefore

$$
H_{1}(v)=\int_{0}^{v} \frac{x d x}{h(x)}
$$

It follows that $H_{1}(v)$ behaves like $\ln (1-v) / h_{2}(1)$ as $v$ approaches one from the left. Substitution for $F$ gives

$$
f^{\prime}=\operatorname{ch}(f) e^{-H_{1}(f)}
$$

and $f$ satisfies the conditions

$$
f(0)=1, \lim _{x \rightarrow \pm \infty} f(x)=0
$$


We need the function $h e^{-H_{1}(f)}$ non-Lipschitz at one in order to have non-uniqueness. From the behaviour of $H_{1}$ near one we find $h(v) e^{-H_{1}(v)}$ behaves like $(1-v)^{1-1 / h_{2}(1)}$ and hence is non-Lipschitz if $h_{2}(1)>1$ or equivalently $h^{\prime}(1)>1$. It is seen that $f(x ; c)=f(c x ; 1)$ and therefore $c$ can be set to one in $(14)$. In this case $f(x ; 1)$ is given implicitly by

$$
\xi=\int_{f(x ; 1)}^{1} \frac{e^{H_{1}(v)} d v}{h(v)} \text { where } H_{1}(v)=\int_{0}^{v} \frac{x d x}{h(x)} .
$$

THEOREM 5. Let $c$ be any positive number, $h(v)=v(v-1) h_{2}(v), h_{2}>0$ in $[0,1], h_{2}(1)>1$. Define

$$
f(x)= \begin{cases}f(c x ; 1) & \text { if } x \leqslant 0 \\ f(-c x ; 1) & \text { if } 0 \leqslant x\end{cases}
$$

where $f(x ; 1)$ is given by $(18)$. Then $u=\gamma^{\prime}(t)+h(f(x-\gamma)) / h^{\prime}(x-\gamma)$ and $u_{x}(x, t)=$ $f(x-\gamma(t))$ where $\gamma$ is any arbitrary function subject only to $\gamma(0)=0$ is the unique traveling wave solution of (13) with $g(t)=\gamma^{\prime}(t)-h(f(\gamma)) / h^{\prime}(\gamma)$.

We illustrate the previous theorem with an example.

ExAmple 1. Choose $h(v)=2(v-1) v$ and $c=1$ thereby satisfying all the requirements of the theorem. Then (13) can be integrated in known hyperbolic trigonometric functions. We get $H_{1}(v)=(1 / 2) \ln (1-v), f(x ; 1)=1 / \cosh ^{2} x$ and therefore $f(x)=1 / \cosh ^{2} x, F(x)=\tanh x$. The soliton solution is $u_{x}(x, t)=1 / \cosh ^{2}(x-\gamma(t))$ and $u(x, t)=\tanh (x-\gamma(t))+d \gamma / d t$. The transformation between $(x, t)$ and $(a, t)$ is given by $\sinh (x-\gamma)=e^{t} \sinh (a)$.

\section{ConClusion}

We have analysed (1) using the method of characteristics. It is found that the problem does not admit shocks and the solution exists for all time. The unique solution is given in Theorem 2 of Section 4 . In Section 3 we derived and found qualitative properties of the zero set of $u(x, t)$. It is found in Section 3 that a novel feature of (1) is that small disturbances propagate with infinite speed. In Section 5 a nonlinear perturbation problem is considered. We proved pointwise convergence of the solution. In Section 6 we showed the existence of a soliton solution to (13).

Subsequently, we have also looked at a generalisation of (1) of the form

$$
\left(\frac{\partial}{\partial t}+u \frac{\partial}{\partial x}\right)\left(\alpha u+u_{x}\right)=0
$$

with $\alpha$ a real number.

This problem may develop a shock in finite time and will be considered in detail elsewhere. 


\section{REFERENCES}

[1] S. Angennent, 'The zero set of a solution of a parabolic equation', J. Reine. Angew. Math. 390 (1988), 79-96.

[2] F. Calogero, 'A solvable nonlinear wave equation', Stud. Appl. Math. (1984), 189-199.

[3] P. Constantin, P.D. Lax and A. Majda, 'A simple one-dimensional model for the three-dimensional vorticity equation', Comm. Pure Appl. Math. 38 (1985), 715-724.

[4] P.G. Drazin, Solitons, London Mathematical Society Lecture Notes Series 85 (Cambridge University Press, Cambridge, New York, 1983).

[5] Y. He and T.B. Moodie, 'A transonic model problem', in Nonlinear diffusion phenomenon, (P.L. Sachdev and R.E. Grundy, Editors) (Norasa Publishing House, New Delhi, 1992), pp. $92-117$.

[6] T.P. Liu, 'Nonlinear resonance for quasilinear hyperbolic equation', J. Math. Phys. 28 (1987), 243-260.

[7] A. Majda, 'Vorticity and the mathematical theory of incompressible fluid flow', Comm. Pure Appl. Math. 39 (1986), S186-S220.

[8] H. Matano, 'Nonincrease of the lap number of a solution for a one dimensional semilinear parabolic equation', J. Fac. Sci. Univ. Tokyo Sect. 1A Math. 29 (1982), 401-411.

[9] S. Schochet, 'Explicit solutions of the viscous model vorticity equation', Comm. Pure Appl. Math. 39 (1986), 531-537.

Department of Mathematics

Monash University

Clayton Vic 3168

Australia

e-mail: sris@wave.maths.monash.edu.au 